\title{
Thyroid Dyshormonogenesis
}

National Cancer Institute

\section{Source}

National Cancer Institute. Thyroid Dyshormonogenesis. NCI Thesaurus. Code C121751.

A defect in any step of the biochemical pathway leading to production of thyroid hormones. 